\title{
Approach to a Message Routing for Wireless Mesh Networks with a Rapidly Changing Topology
}

\author{
Anton S. Churaev ${ }^{1} \&$ Konstantin S. Zaytsev ${ }^{2}$ \\ ${ }^{1}$ Sberbank-Technology Company, Novodanilovskaya naberejnaya st., 10, bldg.3, 117105 Moscow, Russia \\ ${ }^{2}$ National Research Nuclear University MEPhI (Moscow Engineering Physics Institute), Kashirskoe avenue, 31 \\ 115409, Moscow, Russia \\ Correspondence: Konstantin S. Zaytsev, National Research Nuclear University MEPhI (Moscow Engineering \\ Physics Institute), Kashirskoe avenue, 31 115409, Moscow, Russia.
}

Received: July 24, $2014 \quad$ Accepted: August 13, $2014 \quad$ Online Published: October 23, 2014

doi:10.5539/mas.v8n6p178 URL: http://dx.doi.org/10.5539/mas.v8n6p178

\begin{abstract}
A development of the major part of economy branches nowadays is provided through the wide scale adaptation of social networks, which are capable of connecting resources of both legal persons and physical persons. That trend requires a developed infrastructure based on new solutions for the aggregation of mobile devices in a so called wireless mesh networks. One of the central tasks of that aggregation is message routing in networks with a rapidly changing topology. The purpose of the presented paper is an attempt to analyze the main message routing algorithms in wireless networks with respect to mesh networks with rapidly changing topology, and to make recommendations for their improvement and development. As the primary reason, which prevents the achievement of a quality routing in networks with a rapidly changing topology, the instability of links between network nodes is considered. For the assessment of quality of channels between nodes, the implementation of following parameters is proposed: package transmission delay, available network capacity and time of life of a communication channel, which can be easily adjusted by empirically-selectable threshold values. For the solution of the task of message routing of wireless mesh networks with a rapidly changing topology, a criteria is proposed which implements weighted values of proposed parameters. The selection of threshold values of parameters and their weights in order to calculate criterion expression is the important part of a network design, and depends on its expected work characteristics.
\end{abstract}

Keywords: Wireless Mesh Networks (WMN), changing topology, reactive and proactive routing modes, instability of the relations

\section{Introduction}

Wireless Mesh network (WMN) can be perceived in a similar way as a society, in which communication -information transfer is not occurring centrally, but directly from one member of society to another (Bokhari and Zaruba, 2012). A development of routing methods for such networks began comparatively recently, after it was realized that routing algorithms that have been developed for wired networks, are not suitable for that segment of a network market.

All designed routing algorithms are divided into reactive, which lay a route for the package of data from a source node to a destination node only at the time when they send a package (Cisco Guide, 2014), proactive, supporting reachability tables of senders and recipients by permanent background exchanges of information about alterations in a network topology between nodes (Jacquet, 2001), and hybrid (Lumpur, 2012, Lyahov et al., 2012), in which, generally, an initial network is divided into subnets; inside each of them exist proactive algorithms, and interaction between subnets is provided by reactive methods.

In addition, routing algorithms are divided into determined, guaranteeing delivery of data to a recipient, and probabilistic, which ensure delivery of data with some uncertainty.

With the increase of changes of speed in the topology of a network, the effectiveness of the operation of reactive and proactive algorithms is decreasing rapidly. For reactive algorithms, because cached routes of a package transport quickly become obsolete due to the destruction of their constituent links, resulting in a need for the frequent rearrangement of acceptable routes, and as a consequence, to bigger delays in data delivery. For 
proactive algorithms, because of a rapid increase in a service traffic volume, which is designed to support relevance of path tables, and in the end, the collapse of a network because of the exhaustion of the network capability of communication channels.

Nowadays, there are several dozens of routing algorithms for wireless mesh networks, which are adopted by international standards IEEE 802.11 in the form of network protocols or which are appeared as results of purely academic studies (Tripathi J. et al., 2014, Alotaibi E. et al., 2012, Abed A.K. et al., 2014, Jenn-Wei Lin \&Shian-Min Lin., 2014, Wellons J. \& Xue Y., 2014), based on which one can attempt to solve the problem of data packages routing in networks with a rapidly changing topology, basing on a local area network properties consideration, without trying to build a route entirely.

The most appropriate algorithms are OLSR (optimized link state routing) (Clausen, 2003) and its extension QOLSR (QOS optimized link state routing) (Badis, 2005), AODV (ad-hoc on-demand Distance Vector) (Perkins, 2003), ODCR (on-demand Delay-Constrained unicast routing) (Zhang, 2005).

\section{Materials and Methods}

The main idea of proactive OLSR protocol is to minimize the distribution of broadcast messages by reducing the redundant forwarding of messages in borders of one area. When building an acceptable route, each node generates the vicinity of nodes within a single transition: so-called multi point relays (MPR). Neighboring nodes, not included in that vicinity, read and process packages of that node, but does not transmit them further. MPR nodes are selected in the manner that they can cover all the neighboring nodes within two transitions from a node. The smaller set of MPR, the less there is a need to transmit control messages. The protocol of packages routing entirely relies on a selected MPR relays. All packages are sent exactly through these selected nodes and in order to do that each node supports information about the set of its neighbors who have chosen it as MPR. This set is commonly referred as the selector set of multi point repeaters (MPR selector set). In the case of OLSR protocol two types of control messages are used: "Hello" - used to identify neighboring nodes within two transitions and is transmitted in a broadcast way, and "TC (Topology Control)" - used to control a network topology and is periodically sent by each node, in order to form MPR selector set for it. All the information, including pairs $<$ selector of MPR, MPR $>$, is registered in appropriate path tables of nodes. Each contained record has its time of life.

The only factor for the quality assessment of a route is a number of intermediate nodes. The advantage of OLSR protocol is virtually the instant determination of a route, because each of nodes is always has a path table, where routes for any network node are stored. But another side of the determination speed of a route is an increased traffic, which is generated for the transfer of control messages, increased memory requirements for nodes and the fact that routes are always pass through MPR nodes and are not always optimally loaded. In addition, if a route node becomes unavailable, some time passes before the rest of nodes will know about that fact, by means of sending control messages.

QOLSR protocol uses not one, but two indicators to assess the quality of a route for the creation of sets of MPR: a package delay and an available network capability. The evaluation of the delay of a package transmission is carried out statistically, using periodic dispatch of Hello messages. The full delay of package transmission is estimated as the product of an average processing time for one package and a size package queue. An average processing time for one package, in turn, depends on time of a package transmission, and an expected number of repeated transmissions of a package, which depends on the percentage of package loss on MAC level (Media Access Control, hardware address). That percentage of losses is estimated as a number of successfully delivered Hello packages to the total number of Hello packages sent over the certain period of time.

An available network capability is also estimated statistically, considering occupancy of a transfer media, and is calculated as a product of time, when a media is free, and a nominal networking capability.

The clear advantage of that protocol in comparison to OLSR, is the use of two options during the creation of MPR nodes' structure.

Reactive protocol AODV creates routes using cycle "request-response". When an application requests a route to a node, to which there is no route yet in a table, a node in a broadcasting manner sends a request for its search. A node which received that request also in a broadcasting manner sends it further, in the same time recording in its routing table a path up to a source node through a node, from which it received an initial broadcast request. Thus, nodes transmit each other a request, until it reaches a destination node, getting to know about a route to a source node in the same time. If any of intermediate nodes is already aware about a route to a destination node, it transmits a reply to the source of a request, not forwarding its request further. 
Finally, sending node can get several answers, so it is necessary for it to choose a route with the lowest number of intermediate nodes. To reduce amount of control messages and avoid loops, described protocol uses special counters. Each request has a serial number, and intermediate nodes use it, for the purpose of identifying already transferred requests.

The major advantage of AODV protocol is that it does not create an additional traffic in a case there is no data transfer. A search for the best route is determined by a number of intermediate nodes.

Disadvantages of the protocol are the possibility of information loss during crashes processing and during an identification of duplicates of requests serial numbers.

Hybrid distance-vector routing algorithm ODCR is focused on providing routes of data transfer, which are not exceeding a threshold delay time. First, it creates and maintains in a relevant state in nodes of routing table, containing information about the shortest path to each potential destination node. Then, when an application requests a route to a certain node with the condition of a package transmission delay restriction, information from a routing table is used to send a test message to a destination node through the shortest route, in order to check the satisfiability of a route. If a route meets requirements for a maximum possible delay, a destination node responds with ACK package (Acknowledgment). Intermediate nodes, passing ACK message back to a source node, note requirements for the delay of a package and reserve resources at a network level for a following data transfer.

If the shortest route does not meet a determined delay limit, a source node initiates systematic, but limited, a broadcasting process of another route detection.

That algorithm requires additional resources for storage and maintenance of routing tables in a relevant condition, while the objective of systematic process of routes search is to avoid global avalanche dispatch of control messages in order to save resources. In addition, a resource reservation at a network level means that a protocol does not provide clear guarantees for the availability of these resources at the time of data transfer.

In addition to analyzed algorithms there are many other approaches that use for the selection of the optimal route information of transport level, available network capability, temporary delay of package transmission, methods of messages dispatch.

It is clear, that the effective use of a routing algorithm depends on nature of a network, in which it is to be applied, nature of information and additional restrictions on the speed of data transfer, speed of a connection, a need for information streaming, etc. The results of the analysis of routing algorithms are presented in table 1.

Table 1. Comparative characteristics of routing algorithms

\begin{tabular}{|c|c|c|c|}
\hline $\begin{array}{l}\text { Name of } \\
\text { algorithm }\end{array}$ & $\begin{array}{l}\text { Type of } \\
\text { algorithm }\end{array}$ & $\begin{array}{l}\text { Route quality } \\
\text { assessment parameters }\end{array}$ & $\begin{array}{c}\text { Consequences of an implementation in networks with a rapidly } \\
\text { changing topology. }\end{array}$ \\
\hline OLSR & proactive & $\begin{array}{l}-\quad \text { number of } \\
\text { intermediate nodes }\end{array}$ & $\begin{array}{l}\text { A redundant service traffic necessary to maintain routing tables in a } \\
\text { relevant condition is generated }\end{array}$ \\
\hline AODV & reactive & $\begin{array}{l}\text { - number of } \\
\text { intermediate nodes }\end{array}$ & $\begin{array}{l}\text { In a case of a deletion of a channel situated between nodes involved } \\
\text { in a transmission, it is necessary to re-start a route formation } \\
\text { procedure }\end{array}$ \\
\hline QOLSR & proactive & $\begin{array}{l}\text { - a delay of a package } \\
\text { transmission, } \\
\text { - an available network } \\
\text { capability }\end{array}$ & $\begin{array}{l}\text { A redundant service traffic necessary to maintain routing tables in a } \\
\text { relevant condition is generated }\end{array}$ \\
\hline ODCR & $\begin{array}{l}\text { hybrid } \\
\text { (proactive, } \\
\text { reactive) }\end{array}$ & $\begin{array}{l}\text { - a delay in a package } \\
\text { transmission }\end{array}$ & $\begin{array}{l}\text { It is necessary to constantly spend resources to maintain routing } \\
\text { tables }\end{array}$ \\
\hline
\end{tabular}

As it can be seen from the table, the direction of discussed algorithms improvement is to weaken their dependence from rapid changes in the topology of a network. One of the ways to address that problem in the case of a route creation, is to use the local information of nodes, because the maintenance of it in a relevant condition requires the minimum of service messages. In addition, it is clear that a probability of a long route change is higher than the probability of a short route change. 


\section{Results}

Instability of links between nodes is the main reason which is not allowing to achieve a quality routing in networks with a rapidly changing topology, using all algorithms described above. As in the case of a frequent change in a network topology it becomes expensive to maintain information of routing tables in a relevant condition, and, consequently, the implementation of proactive algorithms becomes unreasonable, and, in the case of reactive algorithms, knowledge of an entire route from a sender node to a recipient node becomes unnecessary. An ability to use information of separate local nodes and their neighbors in close vicinity and more careful selection multi point relays (MPR) becomes important.

Considering aforementioned, the methodology of routing becomes locally orientable and requires more careful selection of analyzed parameters of channels and nodes of a network. As a network topology changes rapidly it is necessary to use channel parameters that characterize their stability. One of those kinds of easily calculated parameters is "time of life of a channel" or "age of a channel". Management of routing process settings is conducted by means of heuristic varying of threshold values of selected parameters.

Considering described methodology, we propose one of approaches for a route creation in networks with a rapidly changing topology, continuing to improve OLSR protocol. To obtain necessary information about a network, also two types of service message "Hello" and "TC" will be used. "Hello" message is sent in a broadcasting manner to all available nodes, and further is not relayed by them. Upon receiving this message, a node uses it for the calculation of a channel's quality indicators. A message "TC" is used to inform vicinity nodes of its status and used MPRs.

Now, when assessing the quality of channels between nodes, in addition to parameters that are used in QOLSR protocol (delay of a package transmission; available network capability), the parameter "time of life or age of a channel" will be used. Finally, we have three parameters, defining strategies for routes.

These parameters can be easily adjusted using selected threshold values. The selection of threshold values and their weights in the calculation of criterion expression is the important part of a network design, and it depends on expected characteristics of its operation. In a general case, to calculate a channel characteristic following equation is used:

$$
K=\text { truncate }\left(\frac{\tan ^{-1}\left(T_{l}-T_{l}^{\prime}\right)}{\frac{\pi}{2}}+0.5\right) \cdot\left(N_{t t} \frac{T_{t}^{\prime}}{T_{t}}+N_{v a} \frac{V_{a}}{V_{a}^{\prime}}\right),
$$

where:

$K$ - selection criteria for a node used as MPR;

$T_{l}$ - time of life of a channel;

$T_{l}^{\prime} \quad$ - threshold time of life of a channel;

$T_{t}$ - delay in a package transmission;

$T_{t}^{\prime} \quad$ - threshold delay during a package transmission;

$V_{a}$ - available network capability of a channel;

$V_{a}^{\prime} \quad$ - threshold network capability of a channel;

$N_{t t}$ - weighting factor of a package transmission delay;

$N_{v a}$ - weighting factor of a network capability of a channel.

Calculated in such a way channel characteristic allow to determine the possibility of the implementation of a certain network node as a relay, with this, as it can be seen, all unreliable channels, which time of life of is less than a selected threshold value, can be easily filtered out.

The channel time of life parameter of a channel $\left(T_{l}\right)$ is collected in nodes in a case of receiving Hello message, using a separate counter, which calculates the number of missed messages.

The evaluation of a package transmission delay $\left(T_{t}\right)$ is conducted statistically similar to QOLSR protocol, using periodic distich of Hello messages. The full delay of a package transmission is estimated as the product of the average processing time of one package and packages queue size. The average processing time of a package in 
turn depends on time of a package transmission, and the expected number of repeated transmissions of a package, which depends on the percentage of packages loss at the level of an access control to MAC carrier (Media Access Control). The percentage of losses is estimated as the number of successfully delivered packages Hello to the total number of Hello packages sent over the specific period of time.

The available network capability $\left(V_{a}\right)$ also estimated statistically similar to QOLSR protocol. For that a node monitors occupancy of a transfer media, taking into account periods of contest for an access and periods of collisions. An available network capability is calculated as the product of time, when environment is free, and a nominal networking capability.

In a case, when, after an application of the criteria required number of channels for a message transmission has not been selected (in a general case, there may be a few of them), the selection of missing channels is occurring randomly from the list of unselected channels.

The transmission of a message to "random" nodes is due to a fact that, for nodes, which time of life of channels is less than a threshold, a statistics is not collected, and, therefore, during a selection they are not different from each other.

The broadcast dispatch of messages is also possible, for nodes that do not have sufficient MPR list.

The transmission of a more high-level message conducted as follows. A sender node checks that there is no recipient node among adjacent nodes, if such a node is found; a message is transmitted to it. Otherwise, there is an extended MPR list being formed, which is attached to a message.

Nodes, which received a message for a relay, in the case a message has not been processed before that, verify adjacent nodes for the presence of recipient nodes. In the case of the detection of a destination node the message is sent to it, or the node verifies, whether is it the part for a sender node MPR, or whether is it the part of an extended MPR list for a message, and depending on that, it messages to all nodes, forming a new extended MPR list.

The proposed algorithm allows to achieve the high probability of a message delivery with the additional use of a multiple random walks during the relay of messages through nodes that are not part of MPR.

Major processes of the algorithm are following:

- service processes (sending Hello messages, processing of incoming Hello messages);

- target processes (the dispatch of target messages, relaying messages).

A process in the case of that node generates and sends broadcast messages Hello, has a beginning condition "Event on timer", and an end condition "Sending message". The process activity is presented in figure 1.

As it can be seen from fig.1, when timer is triggered, an increase in message counter is occurring and a new number is assigned to a message. After that happens the update of state tables (table 2) and the dispatch of a broadcast message are occurring.

Considering that state table in the context of that activity, the selection of relay nodes of MPR is conducted as follows. The verification of all records is performed in a state table, for which a difference in time of modification (TM) and current time exceeds a threshold value; for them there is a decrease in time of life of a channel $(T L)$, and at that time of record modification is changed (TM). After modifying a table, the formation of the list of $M P R$ relays in accordance with as elected criterion is occurring.

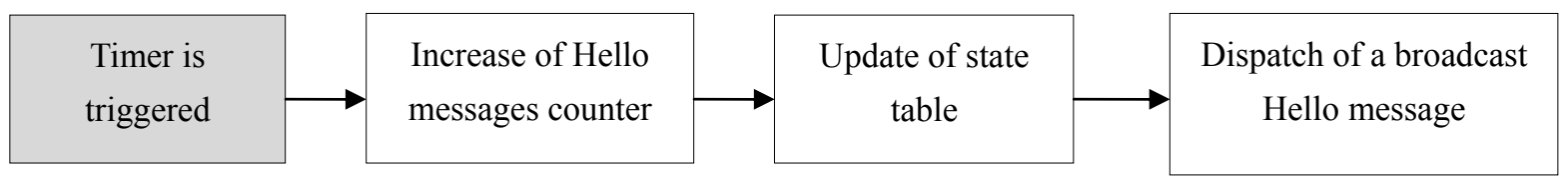

Fig. 1. Activities of process of hello messages dispatch

Upon the completion of a status table update, Hello message is generated, in which an updated value of Hello messages counter is entered, as well as the list of MPR relays. The formed message in a broadcasting manner is transmitted to all nodes.

A process started by an incoming Hello message has a condition for a beginning "Incoming message Hello ", and has the condition of an end "Update corresponding to a sender node in the entry of a state table". The sequence 
of that process activity: receiving Hello message, determining a messages counter delta from that node, updating relevant entries in a state table.

Table 2. The attribute composition of a state table

\begin{tabular}{ll}
\hline Attribute name & Description \\
\hline NN & Unique node ID \\
HN & Counter of Hello message \\
TL & Time of life of a channel \\
TT & Delay in a package transmission \\
VA & Available network capability of a channel \\
TM & Time of an entry modification \\
MPR & Sign of occurrence in the list of MPR relays \\
SMPR & List MPR relays, used by the node.
\end{tabular}

Upon receiving an incoming Hello message, a node on the basis of available information determines the number of not received Hello messages from a sender node, and if a obtained difference is greater than a threshold value, time of life of a channel is reset. After that on the basis of data from a message, as well as its title, corresponding entry in a state table is updated.

The process of messages dispatch has a beginning condition "Request to send messages" and an end condition "Message transmission". The sequence of that process's activities: request for a message dispatch, formation of an extended $M P R$ list, message formation, message transmission.

When a request to send message appears, when the main list of $M P R$ relays is not sufficient to ensure a transmission quality, there is an extended $M P R$ list being formed. After that, there are occur the message formation and the broadcast dispatch of it to all nodes of a network.

The process of a message relaying has a beginning condition "Incoming message", and an end condition "Message transmission". The sequence that process activity is presented in fig. 2 .

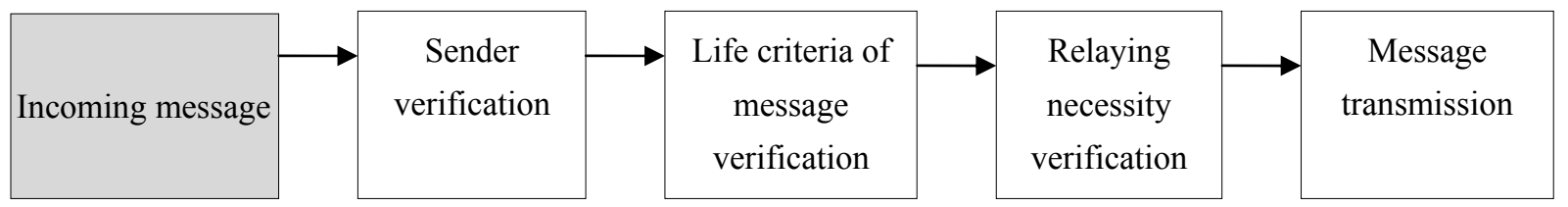

Fig. 2. Activities of messages relaying process

As it can be seen, after receiving an incoming message, a node verifies, whether or not it is the recipient of a message. If it is "yes", there is the processing of an incoming message takes place, otherwise occurs only the verification of life criteria of a message. If life criteria of a message has not been met, it is necessary to delete a message. In meeting conditions of a message life, a node verifies whether it should convey this message further. If a node is located in a relevant $M P R$ list for a sender node, or in an expanded $M P R$ list for an incomingmessage, it relays a message according to its $M P R$ list, or, if necessary, an expanded $M P R$ list formed. At the same time, the message itself can change in the part of life criterion.

Thus, by using the proposed algorithm of packages routing, stable results in networks with a rapidly changing topology can be achieved, using the expanded list of calculated parameters in local vicinities of nodes.

\section{Discussion}

Significant interest in academic society (Bokhari and Zaruba, 2012, Dugaev, 2013, Shamin, 2008) and large IT companies (Cisco Guide, 2014, Meng Fang, 2005) to problems of creation, use and analysis of the effectiveness of modern mesh networks in a recent time is caused by a desire to use private computing resources. Range of studies in that area is gradually shifting from a development and the classification of basic algorithms (Clausen, 2003 \& Zhang, 2005) of routing, applicable in the most networks to the obtainment of private effective solutions (Dugaev, 2013) and modeling of particular networks in order to identify effective routing algorithms (Zacepin, 2013). Wireless mesh networks are now actively being finalized in the context of implementation of various 
topologies (Shamin, 2008), leading companies around the world also offer their designs, but they only use patented closed protocols.

It should be noted, that in the light of requirements' evolution of the major part of mobile users in the direction of large amounts of traffic transfer in real-time, various hybrid protocols are becoming more and more interesting (Lumpur, 2012, Dugaev, 2013, Vázquez-Rodas A. et al., 2014, Di Wu et al., 2013, Gálvez J.J. \& Ruiz P.M., 2013), characteristics of which can be configured for various groups of users.

The proposed approach to routing in mesh networks with a rapidly changing topology is based on ideas of OLSR algorithm (Clausen, 2003) and is aimed at the reduction of dependence from rapid changes in the topology of a network. In order to calculate a generalized characteristics of a channel that algorithm uses threshold values of parameters, such as the delay of a package transmission, an available network capability and time of life of a channel. The problem of the selection of threshold values of parameters, as well as the obtainment of their statistical values requires further research, for example, involving simulation models of networks (Zacepin, 2013).

\section{Conclusion}

The implementation of the proposed algorithm of packages routing allows to obtain stable results in networks with a rapidly changing topology, using the expanded list of calculated parameters in local vicinities of nodes. Conducted systematic analysis of routing protocols in mesh networks with a rapidly changing topology allows to make the following conclusions.

Instability of links between nodes is the main reason for that doesn't allow to achieve better routing in this kind of networks in the case of implementation of popular proactive, reactive and hybrid algorithms.

For the assessment of the quality of channels between nodes it is proposed to use somewhat expanded list of parameters, which includes the delay of packages transmission, an available network capability and time of life of a channel, which can be easily adjusted using empirically-selectable threshold values.

For the calculation of the generalized characteristics of a channel during the solution of a message routing task for wireless mesh networks with a rapidly changing topology a multiplicative criteria introduced that uses weighted values of the proposed parameters. The selection of threshold values of parameters and their weights in the calculation of a criterion expression is the important part of a network design, and it depends on its expected operation characteristics.

\section{References}

Abed, A. K., Oz, G., \& Aybay, I. (2014). Influence of mobility models on the performance of data dissemination and routing in wireless mobile ad hoc networks. Computers \& Electrical Engineering, 40(2), 319-329. http://dx.doi.org/10.1016/j.compeleceng.2013.03.022

Alotaibi, E., \& Mukherjee, B. (2012). A survey on routing algorithms for wireless Ad-Hoc and mesh networks. Computer Networks, 56(2), 940-965. http://dx.doi.org/10.1016/j.comnet.2011.10.011

Badis, H. (2005). QOLSR, QoS routing for ad hoc wireless network using OLSR.Wiley European Trans. Telecommunication, 4(15), 427-442. http://dx.doi.org/10.1002/ett.1067

Bokhari, F., \& Zaruba, G. (2012). The use of smart ants for efficient routing in wireless mesh networks. International Journal of Wireless \& Mobile Networks (IJWMN), 2(4), 113-134.

Cisco Wireless Mesh Access Points, Design and Deployment Guide, Release 7.0. (2014). Retrieved from http://www.cisco.com/c/en/us/td/docs/wireless/technology/mesh/7-0/design/guide/MeshAP_70.html

Clausen, T. (2003). Optimized Link State Routing Protocol (OLSR). Request for Comments: 3626.Date Views 01.04.2014. Retrieved from http://www.ietf.org/rfc/rfc3626.txt

Di, W., Lichun B., Regan, A. C., \& Talcott, C. L. (2013). Large-scale access scheduling in wireless mesh networks using social centrality. Journal of Parallel and Distributed Computing, 73(8), 1049-1065. http://dx.doi.org/10.1016/j.jpdc.2013.03.011

Dugaev, D. (2013). Architecture and hybrid routing algorithm for wireless mesh networks based on the IEEE 802.11S. Digest of XXV international scientific and practical conference "Innovations in science", 11.07.2013, Russia, Novosibirsk. Retrieved from http://sibac.info/10557

Gálvez, J. J., \& Ruiz, P. M. (2013). Efficient rate allocation, routing and channel assignment in wireless mesh networks supporting dynamic traffic flows. Ad Hoc Networks, 11(6), 1765-1781. 
Jacquet, P. (2001). Optimized Link State Routing Protocol for Ad Hoc Networks Proc (pp. 62-68). IEEE Int'l MultiTopic Conf., IEEE Press.

Jenn, W. L., \& Shian, M. L. (2014). A weight-aware channel assignment algorithm for mobile multicast in wireless mesh networks. Journal of Systems and Software, 94, 98-107. http://dx.doi.org/10.1016/j.jss.2014.03.040

Lumpur, K. (2012). IEEE Performance Study of Hybrid Wireless Mesh Protocol (HWMP) for IEEE 802.11s WLAN Mesh Networks. International Conference on Computer and Communication Engineering (ICCCE 2012), 3-5, 48-52.

Lyahov, A. I., \& Nekrasov, P. O., Ostrovski, D. M., Safonov, A. A., \& Horov, E. M. (2012). Analysis of a collective use of proactive and reactive methods of distribution of network information in multi-step wireless networks. Information processes, 3(12), 198-212.

Meng, F. (2005). Routing in Wireless Mesh Networks. ECSE6962. Retrieved from http://www.ecse.rpi.edu/homepages/abouzeid/6962-05/monet22-routing-in-mesh-fang.pdf

Perkins C. (2003). Ad hoc On-Demand Distance Vector (AODV) Routing. Request for Comments: 3561. Retrieved from http://www.ietf.org/html/rfc3561.txt

Shamin P. (2008). Multi-purpose routing in self-organizing networks with limited mobility.Unpublished dissertation in partial fulfilment of the requirements for the degree of Doctor of Philosophy, Vladimir State University, Vladimir.

Tripathi, J., Oliveira, J. C., \& Vasseurb, J. P. (2014). Proactive versus reactive routing in low power and lossy networks: Performance analysis and scalability improvements. Ad Hoc Networks, 23, 121-144. http://dx.doi.org/10.1016/j.adhoc.2014.06.007

Vázquez, R. A., \& Luis J. de la C. L. (2014). A centrality-based topology control protocol for wireless mesh networks. Ad Hoc Networks. In Press, Corrected Proof, Available online 7. Retrieved from http://www.sciencedirect.com/science/article/pii/S1570870514001577

Wellons, J., \& Xue, Y. (2014). The robust joint solution for channel assignment and routing for wireless mesh networks with time partitioning. Ad Hoc Networks, 13(Part A), 210-221. http://dx.doi.org/10.1016/j.adhoc.2011.05.002

Zacepin, E. (2013). An overview of the features of routing protocols in mesh networks. International Journal Experimental Education, 10(part 2), 342-345. Retrieved from http://www.rae.ru/meo/?section=content\&op=show_article\&article_id $=4243$

Zhang, B. (2005). QoS routing for wireless ad hoc networks: problems, algorithms and protocols. Communications Magazine, IEEE, 43(10), 110-117. http://dx.doi.org/10.1109/ MCOM.2005.1522133

\section{Copyrights}

Copyright for this article is retained by the author(s), with first publication rights granted to the journal.

This is an open-access article distributed under the terms and conditions of the Creative Commons Attribution license (http://creativecommons.org/licenses/by/3.0/). 